\title{
Solving guided wave modes in plasmonic crystals
}

\author{
Chien C. Chang, ${ }^{1,2,3, *}$ Yu-Chen Shu, ${ }^{1,3}$ and I-Liang Chern ${ }^{3,4}$ \\ ${ }^{1}$ Division of Mechanics, Research Center for Applied Sciences, Academia Sinica, Taipei 115, Taiwan, Republic of China \\ ${ }^{2}$ Institute of Applied Mechanics, National Taiwan University, Taipei 106, Taiwan, Republic of China \\ ${ }^{3}$ Taida Institute of Mathematical Sciences, National Taiwan University, Taipei 106, Taiwan, Republic of China \\ ${ }^{4}$ Department of Mathematics, National Taiwan University, Taipei 106, Taiwan, Republic of China
}

(Received 1 May 2008; published 30 July 2008)

\begin{abstract}
In this study, we present an interfacial operator approach for solving guided wave modes of plasmonic crystals. They are formulated as an eigenvalue problem of the wave number along the axis of the crystal. In this formulation, the permittivity and permeability of the metallic component can be arbitrary functions of frequency. Moreover, a coupling interface method is introduced to facilitate accurate treatment of the interface conditions with an arbitrary shape between the metal and host materials. Numerical results are illustrated for different shapes of plasmonic crystals, and physical significance is discussed.
\end{abstract}

DOI: 10.1103/PhysRevB.78.035133

PACS number(s): 73.20.Mf, 02.70.-c, 78.20.Bh

\section{INTRODUCTION}

Plasmonics is considered to be the strongest interplay of both optical and electronic data transfer along a tiny metal. ${ }^{1}$ It has found important applications in modern nanotechnology such as magneto-optic data storage, microscopy, solar cells, sensors for detecting biological molecules, and plasmonic crystals. ${ }^{2}$ However, it has been difficult to solve eigenmodes of plasmonic crystals in two or three dimensions either analytically or numerically. This is mainly because the metal is dispersive in frequency and the underlined equation changes its type from being elliptic (metal) to hyperbolic (host medium). When the equation is solved numerically, the change of type makes the discrete systems nondefinite (neither positive definite nor negative definite). Moreover, plasmonic modes may be highly localized, rendering themselves another difficulty to be resolved numerically.

In an early study, ${ }^{3}$ we proposed the method of interfacial operator to solve transverse magnetic (TM) eigenmodes of plasmonic crystals in two dimensions. The method was subsequently applied to solve planar eigenmodes of periodic arrays of polar materials ${ }^{4}$ and of negative-index materials. ${ }^{5}$ In these previous studies, we considered solving eigenmodes as an eigenvalue problem of the frequency, and had to exploit the specific structures of the dielectric functions of the metallic and polar materials. In the present study, the method of interfacial operator is reformulated to solve for guided wave modes in plasmonic crystals. Solving guided wave modes is now considered as an eigenvalue problem for the wave number (rather than the frequency) along the axis of the crystal. The newly formulated method presents a breakthrough by allowing the permittivity and permeability of the metal or polar materials to be arbitrary functions of frequency. Moreover, plasmonic waveguides and band structures may depend significantly on the shape of the interface between the metal and host medium. Therefore, we apply the coupling interface method, developed by Chern and Shu, ${ }^{6}$ to accurately treat a general shape of interface. Furthermore, it must be also noted that unlike the previous studies for two-dimensional problems, the plasmonic guided wave modes are in general hybrid transverse electric (TE) and TM modes, because the electric and magnetic fields are coupled through the interface conditions.

There are a few other methods which have been applied to solve for the eigenmodes of plasmonic crystals. The method of plane-wave expansion ${ }^{7}$ is most commonly used. Other approaches such as transfer-matrix method ${ }^{8}$ multiple-scattering theory or Korringa-Kohn-Rostoker (KKR) method, ${ }^{9-11}$ layerKKR method, ${ }^{12}$ multiple multipole method, ${ }^{13}$ and finitedifference time-domain method ${ }^{14}$ can also be applied to frequency-dependent problems. The method of multiple multipole expansion ${ }^{13}$ is one choice in which a careful definition of cost function is required for detecting resonant frequencies. Also, we have seen the method of vectorial eigenmode expansion applied to study phonon polariton excitation in photonic crystals. ${ }^{15}$ The present approach, the interfacial operator together with the coupling interface method, can directly solve for the plasmonic guided wave modes with accurate treatment of arbitrary-shaped interface between the metal and host medium.

\section{EQUATIONS FOR WAVEGUIDES}

Consider waveguides along the axis of two-dimensional crystals. The crystal geometry is assumed to be periodic in the $x-y$ plane with a unit cell shown schematically in Fig. 1 .

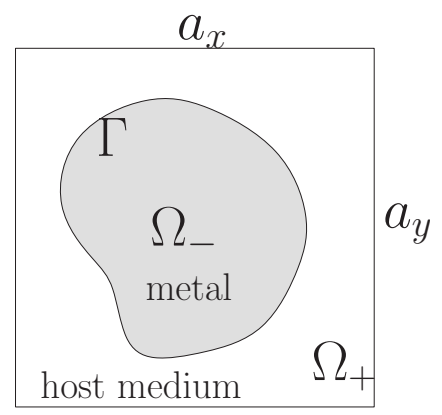

FIG. 1. The unit cell of the plasmonic crystal in the $x-y$ plane. $a_{x}$ and $a_{y}$ are the lengths of the unit cells in the $x$ and $y$ directions. $\Gamma$ is the interface between two different materials with $\Omega_{-}$(metal) and $\Omega_{+}$(host medium). 
The guided modes are in general hybrid modes, and may be simplified into TE and TM modes only under certain circumstances. For generality, we start with hybrid modes. Since the waveguide is homogeneous in the $z$ direction, we look for monochromatic modes of the form:

$$
\begin{aligned}
\mathbf{E}(x, y, z, t) & =\left(E_{x}, E_{y}, E_{z}\right) e^{i(k z-\omega t)}, \\
\mathbf{H}(x, y, z, t) & =\left(H_{x}, H_{y}, H_{z}\right) e^{i(k z-\omega t)},
\end{aligned}
$$

where $\omega$ is the frequency; $k$ is the wave number along the $z$ direction (axial wave number); $\left(E_{x}, E_{y}, E_{z}\right)$ and $\left(H_{x}, H_{y}, H_{z}\right)$ are functions of $(x, y)$. Substituting Eqs. (1) and (2) into Faraday's and Ampére's laws yields the following equations for $E_{z}$ and $H_{z}$ :

$$
\begin{gathered}
\nabla \cdot\left(\frac{\omega \varepsilon_{0} \varepsilon \nabla E_{z}}{M}\right)+\nabla \times\left(\frac{k \nabla H_{z}}{M}\right)=-\omega \varepsilon_{0} \varepsilon E_{z}, \\
\nabla \cdot\left(\frac{\omega \mu_{0} \mu \nabla H_{z}}{M}\right)-\nabla \times\left(\frac{k \nabla E_{z}}{M}\right)=-\omega \mu_{0} \mu H_{z},
\end{gathered}
$$

where we have defined $M=(\omega / c)^{2} \varepsilon \mu-k^{2}$. Note that $c$ is the speed of light in vacuum, $\varepsilon_{0}$ and $\mu_{0}$ are the permittivity and permeability constants in vacuum, and $\varepsilon$ and $\mu$ are the relative permittivity and permeability, respectively. Notice that they are frequency-dependent functions. The symbol $\nabla$ denotes $\left(\frac{\partial}{\partial x}, \frac{\partial}{\partial y}\right)$. Let $\Gamma$ be the interface between two different materials, which separates the domain into two subdomains $\Omega_{+}$(host medium) and $\Omega_{-}$(metal) (Fig. 1). On $\Gamma$, the following interface conditions should be satisfied:

$$
\begin{gathered}
{\left[E_{z}\right]_{\Gamma}=0,} \\
{\left[H_{z}\right]_{\Gamma}=0,} \\
\omega\left[\frac{\varepsilon_{0} \varepsilon}{M} \frac{\partial E_{z}}{\partial n}\right]_{\Gamma}=-k\left[\frac{1}{M} \frac{\partial H_{z}}{\partial s}\right]_{\Gamma} \\
\omega\left[\frac{\mu_{0} \mu}{M} \frac{\partial H_{z}}{\partial n}\right]_{\Gamma}=k\left[\frac{1}{M} \frac{\partial E_{z}}{\partial s}\right]_{\Gamma},
\end{gathered}
$$

where $n$ and $s$ are unit normal and tangential vectors at the interface, respectively, and $[u]_{\Gamma}$ denotes the difference of $u$ between $\Omega_{+}$and $\Omega_{-}$sides at the interface $\Gamma$. These interface conditions can be derived directly from Faraday's and Ampére's laws. On the cell boundary, the Bloch conditions are applied:

$$
\begin{aligned}
& \mathbf{E}\left(x+a_{x}, y+a_{y}\right)=e^{i\left(k_{x} a_{x}+k_{y} a_{y}\right)} \mathbf{E}(x, y), \\
& \mathbf{H}\left(x+a_{x}, y+a_{y}\right)=e^{i\left(k_{x} a_{x}+k_{y} a_{y}\right)} \mathbf{H}(x, y),
\end{aligned}
$$

where $a_{x}$ and $a_{y}$ are the side lengths of the cell in the $x$ and $y$ directions, and $\left(k_{x}, k_{y}\right)$ is the Bloch wave vector.

\section{METHOD OF INTERFACIAL OPERATOR}

Due to the fact that $\varepsilon$ and $\mu$ are frequency-dependent functions, we shall solve the problem of plasmonic guided wave modes as an eigenvalue problem for $k$ with given $\omega$. In the interior of each region, the permittivity and permeability are spatially independent, thus Eqs. (3) and (4) are reduced to the Helmholtz equations with constant coefficients:

$$
\begin{aligned}
& {\left[\nabla^{2}+\left(\frac{\omega}{c}\right)^{2} \varepsilon \mu\right] E=k^{2} E,} \\
& {\left[\nabla^{2}+\left(\frac{\omega}{c}\right)^{2} \varepsilon \mu\right] H=k^{2} H .}
\end{aligned}
$$

Here the subindex $z$ is dropped for notational simplicity. In order to express the interface conditions as an eigenvalue problem like Eqs. (11) and (12), we introduce two auxiliary interface variables:

$$
\begin{gathered}
J_{E}=\left[\varepsilon \frac{\partial E}{\partial n}\right]_{\Gamma}, \\
J_{H}=\left[\mu \frac{\partial H}{\partial n}\right]_{\Gamma},
\end{gathered}
$$

and rearrange Eqs. (7) and (8) as

$$
\begin{aligned}
& \Lambda\left(\left[\frac{1}{\mu} \frac{\partial E}{\partial n}\right]_{\Gamma}+\frac{k}{\omega \varepsilon_{0}}\left[\frac{1}{\varepsilon \mu} \frac{\partial H}{\partial s}\right]_{\Gamma}\right)=k^{2} J_{E}, \\
& \Lambda\left(\left[\frac{1}{\varepsilon} \frac{\partial H}{\partial n}\right]_{\Gamma}-\frac{k}{\omega \mu_{0}}\left[\frac{1}{\varepsilon \mu} \frac{\partial E}{\partial s}\right]_{\Gamma}\right)=k^{2} J_{H},
\end{aligned}
$$

with the help of Eqs. (5) and (6). Here $\Lambda=\left(\frac{\omega}{c}\right)^{2} \varepsilon_{+} \varepsilon_{-} \mu_{+} \mu_{-}$and $\varepsilon_{ \pm}$and $\mu_{ \pm}$are the relative permittivity and permeability functions in $\Omega_{ \pm}$, respectively. The left-hand side of Eqs. (15) and (16) will be called the interfacial operators. It will be re-expressed in terms of $E, H, J_{E}$, and $J_{H}$ in the later section during discretization procedure. Equations (11), (12), (15), and (16), together with the Bloch boundary condition, constitute a quadratic eigenvalue problem for $k$ with unknowns $\left(E, H, J_{E}\right.$, and $\left.J_{H}\right)$, since both $k$ and $k^{2}$ appear in Eqs. (15) and (16).

\section{COUPLING INTERFACE METHOD}

To accurately discretize the above equations, we adopt the coupling interface method. ${ }^{6}$ This is a second-order accurate finite-difference method under Cartesian grid, and it treats the interface as an immersed boundary. The advantages of this method include that: (1) it is a dimension-by-dimension approach, thus has no limitation on the dimensionality; (2) it has little constraint on the geometry of the interfaces. Further, this method uses only first-order derivatives on the interface, and is thus natural to incorporate with the interfacial operator approach, which introduces first-order derivatives as auxiliary variables. Thus, we explain the method in detail for one-dimensional case and sketch the idea in two dimensions.

\section{A. One-dimensional layer structure}

We consider a periodic structure of parallel slabs (Fig. 2). The propagation direction is in the $z$ direction and thus the 


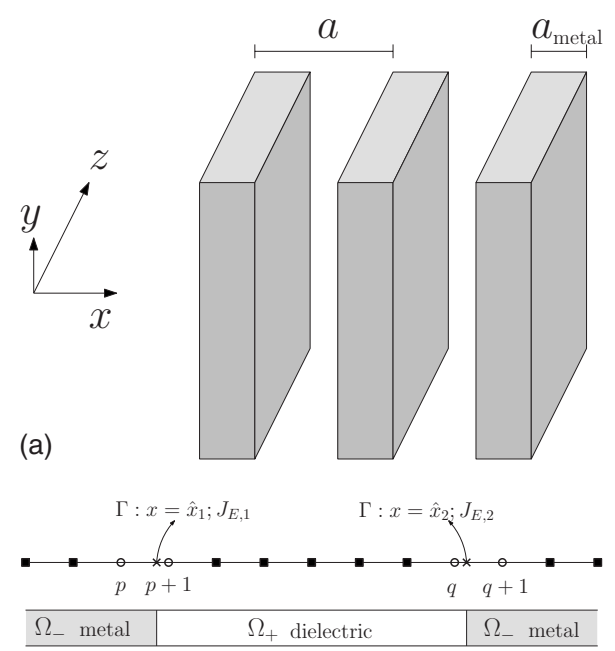

(b)

FIG. 2. (a) A layer structure wave guide. The wave guide consists of metal and air. The gray parts are metal. We assume that the length of a unit cell is $a$ and the length of metal is $a_{\text {metal }}$. (b) The discretization near the interface $\Gamma$ between metal and dielectric. The square points are those grid points in the strict insides of the metal and host medium. The circle points are the grid points near the interface. We assume that $\hat{x}_{1}$ is in the cell $\left[x_{p}, x_{p+1}\right]$ and $\hat{x}_{2}$ is in the cell $\left[x_{q}, x_{q+1}\right]$. The interfacial variables $J_{E, 1}$ and $J_{E, 2}$ are defined at $\hat{x}_{1}$ and $\hat{x}_{2}$, respectively.

fields $E$ and $H$ are constants along the $y$ direction. In this case, $\frac{\partial E}{\partial s}=\frac{\partial E}{\partial y}=0$ and $\frac{\partial H}{\partial s}=\frac{\partial H}{\partial y}=0$ in the interface conditions (15) and (16). Thus, the $E$ and $H$ fields are decoupled. The guided waves can be decomposed as a combination of TM mode $(H \equiv 0)$ and TE mode $(E \equiv 0)$. We shall discuss the discretization for TM mode below. The TE mode can be treated similarly.

For TM modes, $E$ is only a function of $x$. Equation (11), the interface conditions (5) and (7), and the Bloch condition can be simplified as:

$$
\begin{gathered}
E^{\prime \prime}+\left(\frac{\omega}{c}\right)^{2} \varepsilon \mu E=k^{2} E, \\
{[E]_{\Gamma}=0,} \\
\Lambda\left[\frac{1}{\mu} E^{\prime}\right]_{\Gamma}=k^{2} J_{E}, \text { with } J_{E}:=\left[\varepsilon E^{\prime}\right]_{\Gamma}, \\
E(x+a)=e^{i k_{x} a} E(x) .
\end{gathered}
$$

Here $E^{\prime}$ denotes $\frac{d}{d x} E$.

To discretize these equations, the unit cell $[0, a]$ is partitioned uniformly into $N$ subintervals with size $h=\frac{a}{N}$. Let us denote $x_{i}=i h$ and $E_{i}=E\left(x_{i}\right), i=1, \ldots, N$. There are two interface points $x=\hat{x}_{1}$ and $\hat{x}_{2}$ and the interfacial variables $J_{E, 1}$ and $J_{E, 2}$ are defined on them (Fig. 2). Suppose they lie in $\left[x_{p}, x_{p+1}\right)$ and $\left[x_{q}, x_{q+1}\right)$, respectively, for some integers $p$ and $q$. At an interior grid point $x_{i}$ (including periodic boundary), the standard central finite-difference method is adopted for Eq. (17):

$$
\frac{1}{h^{2}}\left(E_{i-1}-2 E_{i}+E_{i+1}\right)+\left(\frac{\omega}{c}\right)^{2} \varepsilon_{i} \mu_{i} E_{i}=k^{2} E_{i}+O\left(h^{2}\right) .
$$

At the grid points $x_{p}, x_{p+1}, x_{q}, x_{q+1}$, we perform the following discretization. We shall only need to explain the case for the pair $x_{p}$ and $x_{p+1}$. The other case is the same. In the neighborhood of $\hat{x}_{1}$, we approximate $E(x)$ by quadratic functions $E_{ \pm}(x)$ in the region $\Omega_{ \pm}$, respectively:

$$
\begin{aligned}
E_{-}(x)= & E_{p}+\frac{1}{h}\left(E_{p}-E_{p-1}\right)\left(x-x_{p}\right)+\frac{E_{p}^{\prime \prime}}{2 h^{2}}\left(x-x_{p}\right) \\
& \times\left(x-x_{p-1}\right)+O\left(h^{3}\right), \\
E_{+}(x)= & E_{p+1}+\frac{1}{h}\left(E_{p+2}-E_{p+1}\right)\left(x-x_{p+1}\right) \\
& +\frac{E_{p+1}^{\prime \prime}}{2 h^{2}}\left(x-x_{p+1}\right)\left(x-x_{p+2}\right)+O\left(h^{3}\right) .
\end{aligned}
$$

By applying the interface conditions $[E]_{\hat{x}_{1}}=0$ and $\left[\varepsilon E^{\prime}\right]_{\hat{x}_{1}}$ $=J_{E, 1}$ in the above two equations, we can express $E_{p}^{\prime \prime}$ and $E_{p+1}^{\prime \prime}$ as linear combinations of $E_{p-1}, E_{p}, E_{p+1}, E_{p+2}$ and $J_{E, 1}$ :

$$
\begin{gathered}
E_{p}^{\prime \prime}=\mathcal{L}_{p}\left(E_{p-1: p+2}, J_{E, 1}\right)+O(h), \\
E_{p+1}^{\prime \prime}=\mathcal{L}_{p+1}\left(E_{p-1: p+2}, J_{E, 1}\right)+O(h),
\end{gathered}
$$

where $E_{p-1: p+2}$ is an abbreviation of $\left(E_{p-1},, E_{p+2}\right)$. The notation $p-1: p+2$ represents the index running from $p-1$ to $p+2$. Thus, we arrive at a finite-difference approximation for Eq. (17) at $x_{p}$ and $x_{p+1}$ :

$$
\begin{gathered}
\mathcal{L}_{p}\left(E_{p-1: p+2}, J_{E, 1}\right)+\left(\frac{\omega}{c}\right)^{2} \varepsilon_{p} \mu_{p} E_{p}=k^{2} E_{p}+O(h), \\
\mathcal{L}_{p+1}\left(E_{p-1: p+2}, J_{E, 1}\right)+\left(\frac{\omega}{c}\right)^{2} \varepsilon_{p+1} \mu_{p+1} E_{p+1}=k^{2} E_{p+1}+O(h) .
\end{gathered}
$$

Finally we need to discretize Eq. (19) for the auxiliary variable $J_{E, 1}$. We re-express $\left[\frac{1}{\mu} E^{\prime}\right]_{\hat{x}_{1}}$ in terms of $J_{E, 1}$ as follows:

$$
\left[\frac{1}{\mu} E^{\prime}\right]_{\hat{x}_{1}}=\frac{1}{\varepsilon_{+} \mu_{+}} J_{E, 1}+\frac{\varepsilon_{-} \mu_{-}-\varepsilon_{+} \mu_{+}}{\varepsilon_{+} \mu_{+} \mu_{-}} E_{-}^{\prime}\left(\hat{x}_{1}\right),
$$

and $E_{-}^{\prime}\left(\hat{x}_{1}\right)$ in turn can be approximated by:

$$
E_{-}^{\prime}\left(\hat{x}_{1}\right)=\frac{E_{p}-E_{p-1}}{h}+\left(\frac{1}{2}+\alpha\right) h E_{p}^{\prime \prime}+O\left(h^{2}\right),
$$

where $\alpha=\left(\hat{x}_{1}-x_{p}\right) / h$. Combining Eqs. (24), (28), and (29), the interfacial operator in Eq. (19) can be approximated by a linear combination of $\left(E_{p-1: p+2}, J_{E, 1}\right)$ and the interface condition can be expressed as

$$
\mathcal{J}\left(E_{p-1: p+2}, J_{E, 1}\right)=k^{2} J_{E, 1}+O\left(h^{2}\right),
$$

where $\mathcal{J}$ is a linear function.

By omitting the truncation error terms, Eqs. (21), (26), (27), and (30) form a standard eigenvalue problem: 


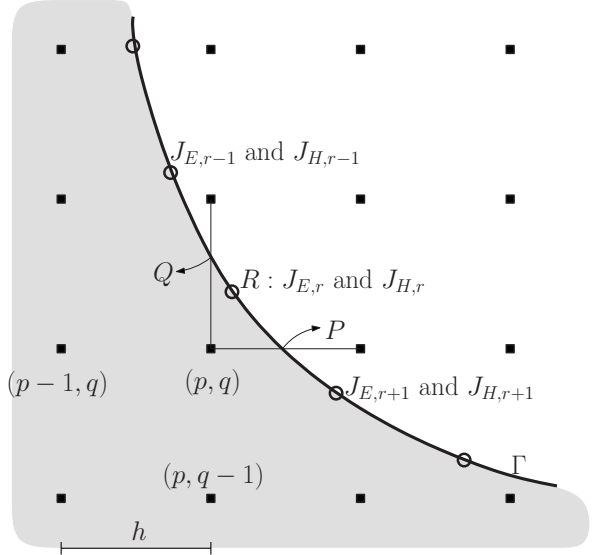

FIG. 3. Square: Numerical Cartesian grid. Circle: Interfacial operators. $J_{E, r-1: r+1}$ and $J_{H, r-1: r+1}$ are interfacial variables. $n$ and $s$ are the unit normal and tangent vectors at $R$. The gray area is $\Omega_{-}$and the white area is $\Omega_{+}$.

$$
\mathbf{A}_{\mathrm{TM}} \mathbf{U}_{\mathrm{TM}}=k^{2} \mathbf{U}_{\mathrm{TM}},
$$

where $\quad \mathbf{A}_{\mathrm{TM}}$ is a $(N+2) \times(N+2)$ matrix and $\mathbf{U}_{\mathrm{TM}}$ $=\left[\bar{E}_{1: N}, \bar{J}_{E, 1}, \bar{J}_{E, 2}\right]^{T} . \bar{E}_{1: N}, \bar{J}_{E, 1}$, and $\bar{J}_{E, 2}$ are the numerical solutions of $E_{1: N}, J_{E, 1}$, and $J_{E, 2}$.

\section{B. Two-dimensional structure}

For two-dimensional guided wave problems, the $E$ and $H$ fields are in general coupled through the interface conditions (7) and (8). Therefore, we need to solve both Eqs. (11) and (12) simultaneously.

To discretize these equations, we partition the unit cell $[0, a] \times[0, a]$ uniformly into $N^{2}$ squares with mesh size $h$ $=\frac{a}{N}$. The Cartesian grid points are $\left(x_{i}, y_{j}\right),(i h, j h), 1 \leq i, j$ $\leq N$. The abbreviation $E_{i, j}=E\left(x_{i}, y_{j}\right)$ is used. We shall also abbreviate the unknowns $\left(E_{1,1}, \cdots, E_{N, N}\right)$ by $E_{1: N, 1: N}$. On the interface $\Gamma$, a set of uniformly distributed grids based on arc length on $\Gamma$ is adopted. They are labeled by $\left(\hat{x}_{r}, y_{r}\right), r$ $=1, \cdots, N_{\mathrm{J}}$. At these points, the auxiliary interface variables $\left(J_{E, r}, J_{H, r}\right)$ are defined.

\section{Discretize the equations at Cartesian grids}

In an interior Cartesian grid point, we discretize Eqs. (11) and (12) by the standard central finite-difference method. At a Cartesian grid point which is adjacent to the interface, the following coupling interface method is adopted. We illustrate the idea for the case in Fig. 3, where $\Gamma$ intersects both $x$ and $y$ axes. In Fig. 3, $\left(x_{p}, y_{q}\right)$ is the grid point at which we want to derive a finite-difference equation; $P$ and $Q$ are the intersections of $\Gamma$ and the $x$ and $y$ axes from $\left(x_{p}, y_{q}\right)$; and $R$ $=\left(\hat{x}_{r}, \hat{y}_{r}\right)$ is the closest interface grid point to $\left(x_{p}, y_{q}\right)$. Our goal is to derive a finite-difference approximation for

$$
\nabla^{2} E=\left.\frac{\partial^{2} E}{\partial x^{2}}\right|_{p, q}+\left.\frac{\partial^{2} E}{\partial y^{2}}\right|_{p, q},
$$

$$
\nabla^{2} H=\left.\frac{\partial^{2} H}{\partial x^{2}}\right|_{p, q}+\left.\frac{\partial^{2} H}{\partial y^{2}}\right|_{p, q},
$$

in terms of the grid data and the interfacial variables. The discretization procedure is divided into the following steps.

(a) Dimension-by-dimension approach. We apply the previous one-dimensional method in both $x$ and $y$ direction separately. Namely, along the grid line $y=y_{q}$, we approximate $\left.\frac{\partial^{2} E}{\partial x^{2}}\right|_{p, q}$ and $\left.\frac{\partial^{2} E}{\partial x^{2}}\right|_{p+1, q}$ in terms of $E_{p-1: p+2, q}$ and $\left[\varepsilon \frac{\partial E}{\partial x}\right]_{P}$. Notice that our interfacial variables are defined at $R$, not at $P$ nor $Q$. Thus, we make slight modification, i.e. replacing $\left[\varepsilon \frac{\partial E}{\partial x}\right]_{P}$ by $\left[\varepsilon \frac{\partial E}{\partial x}\right]_{\left(\hat{x}_{r}, y_{q}\right)}$ in the derivation, of our one dimensional approach and the resulting formula for $\left.\frac{\partial^{2} E}{\partial x^{2}}\right|_{p, q}$ changes a little bit. We arrive at the formula

$$
\left.\frac{\partial^{2} E}{\partial x^{2}}\right|_{p, q}=\mathcal{L}_{E_{x x}}\left(E_{p-1: p+2, q},\left[\varepsilon \frac{\partial E}{\partial x}\right]_{\left(\hat{x}_{r}, y_{q}\right)}\right)+O(h) .
$$

Similarly, we get

$$
\left.\frac{\partial^{2} E}{\partial y^{2}}\right|_{p, q}=\mathcal{L}_{E_{y y}}\left(E_{p, q-1: q+2},\left[\varepsilon \frac{\partial E}{\partial y}\right]_{\left(x_{p}, \hat{y}_{r}\right)}\right)+O(h) .
$$

(b) Using the interfacial variables at interface grid points. We express Eqs. (32) and (33) in terms of the interface data at $R$ :

$$
\begin{aligned}
& {\left[\varepsilon \frac{\partial E}{\partial x}\right]_{\hat{x}_{r}, y_{q}} \approx\left[\varepsilon \frac{\partial E}{\partial x}\right]_{R}+\left(y_{q}-\hat{y}_{r}\right)\left[\varepsilon \frac{\partial^{2} E}{\partial x \partial y}\right]_{R},} \\
& {\left[\varepsilon \frac{\partial E}{\partial y}\right]_{x_{p}, \hat{y}_{r}} \approx\left[\varepsilon \frac{\partial E}{\partial y}\right]_{R}+\left(x_{p}-\hat{x}_{r}\right)\left[\varepsilon \frac{\partial^{2} E}{\partial x \partial y}\right]_{R} .}
\end{aligned}
$$

The jump of the cross derivative term in Eqs. (34) and (35) is expanded and approximated by

$$
\begin{gathered}
{\left[\varepsilon \frac{\partial^{2} E}{\partial x \partial y}\right]_{R}=\left.\varepsilon_{+} \frac{\partial^{2} E_{+}}{\partial x \partial y}\right|_{R}-\left.\varepsilon_{-} \frac{\partial^{2} E_{-}}{\partial x \partial y}\right|_{R}} \\
\left.\frac{\partial^{2} E_{+}}{\partial x \partial y}\right|_{R} \approx \frac{1}{h^{2}}\left(E_{p+2, q+2}+E_{p+1, q+1}-E_{p+2, q+1}-E_{p+1, q+2}\right) \\
\left.\frac{\partial^{2} E_{-}}{\partial x \partial y}\right|_{R} \approx \frac{1}{h^{2}}\left(E_{p, q}+E_{p-1, q-1}-E_{p, q-1}-E_{p-1, q}\right)
\end{gathered}
$$

The truncation error of the above approximation is $O(h)$. Next, we re-express the terms $\left[\varepsilon \frac{\partial E}{\partial x}\right]_{R}$ and $\left[\varepsilon \frac{\partial E}{\partial y}\right]_{R}$ in Eqs. (34) and (35) in terms of normal and tangential derivatives of $E$ at $R$ :

$$
\left[\varepsilon \frac{\partial E}{\partial x}\right]_{R}=n_{x} J_{E, r}+s_{x}\left(\left.[\varepsilon]_{R} \frac{\partial E_{-}}{\partial s}\right|_{R}+\left[\frac{\partial E}{\partial s}\right]_{R}\right),
$$




$$
\left[\varepsilon \frac{\partial E}{\partial y}\right]_{R}=n_{y} J_{E, r}+s_{y}\left(\left.[\varepsilon]_{R} \frac{\partial E_{-}}{\partial s}\right|_{R}+\left[\frac{\partial E}{\partial s}\right]_{R}\right),
$$

where $n=\left(n_{x}, n_{y}\right)$ and $s=\left(s_{x}, s_{y}\right)$ are the unit normal and tangential vectors of $\Gamma$ at $R$, respectively. Due to $[E]_{R}=0$, we have

$$
\left[\frac{\partial E}{\partial s}\right]_{R}=0 .
$$

(c) Interpolation for the one-side tangential derivative. The one-side tangential derivative can be expressed as

$$
\left.\frac{\partial E_{-}}{\partial s}\right|_{R}=\left.s_{x} \frac{\partial E_{-}}{\partial x}\right|_{R}+\left.s_{y} \frac{\partial E_{-}}{\partial y}\right|_{R} .
$$

The one-side partial derivatives at $R$ can be approximated by

$$
\begin{aligned}
\left.\frac{\partial E_{-}}{\partial x}\right|_{R} \approx & \frac{1}{h}\left(E_{p, q}-E_{p-1, q}\right)+\left.\left(\frac{1}{2}+\alpha_{x}\right) h \frac{\partial^{2} E}{\partial x^{2}}\right|_{p, q} \\
& +\left.\alpha_{y} h \frac{\partial^{2} E}{\partial x \partial y}\right|_{p, q}, \\
\left.\frac{\partial E_{-}}{\partial y}\right|_{R} \approx & \frac{1}{h}\left(E_{p, q}-E_{p, q-1}\right)+\left.\left(\frac{1}{2}+\alpha_{y}\right) h \frac{\partial^{2} E}{\partial y^{2}}\right|_{p, q} \\
& +\left.\alpha_{x} h \frac{\partial^{2} E}{\partial x \partial y}\right|_{p, q},
\end{aligned}
$$

where $\alpha_{x}=\left(\hat{x}_{r}-x_{p}\right) / h$ and $\alpha_{y}=\left(\hat{y}_{r}-y_{q}\right) / h$. The cross derivative can be approximated by

$$
\left.\frac{\partial^{2} E}{\partial x \partial y}\right|_{p, q} \approx \frac{1}{h^{2}}\left(E_{p, q}-E_{p-1, q}-E_{p, q-1}+E_{p-1, q-1}\right) .
$$

After above three steps, we deduce a coupling equation for the second-order partial derivatives $\left.\frac{\partial^{2} E}{\partial x^{2}}\right|_{p, q}$ and $\left.\frac{\partial^{2} E}{\partial y^{2}}\right|_{p, q}$. This coupling equation is solvable practically. Finally, we get

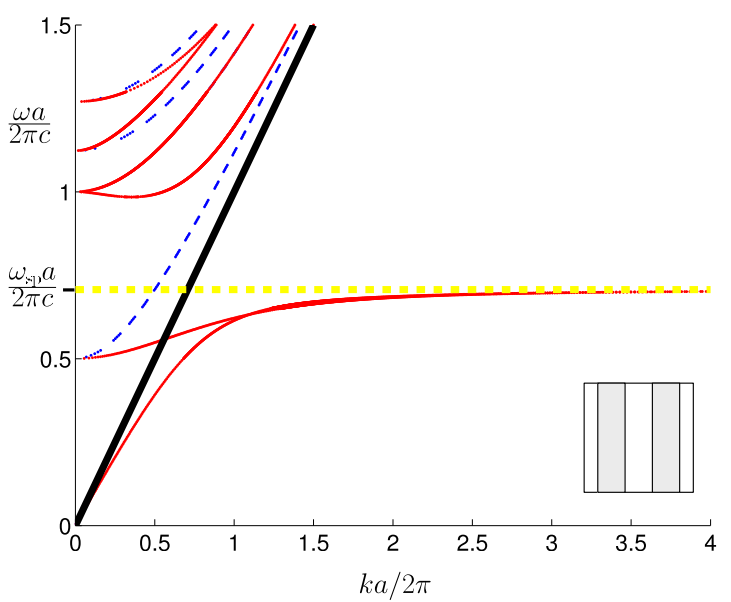

FIG. 4. (Color online) The frequency $\omega$ versus axial wave number $k$ for the layer structure. The Bloch wave number is $k_{x}=0$. The filling ratio of metal is 0.4. $a$ is the length of the unit cell. The red (gray) line is TM mode and the blue (dark gray) dash line is TE mode. The black line is the light line. The yellow (light gray) dash line is the line $\omega=\omega_{\mathrm{sp}}$, the surface-plasmon frequency.
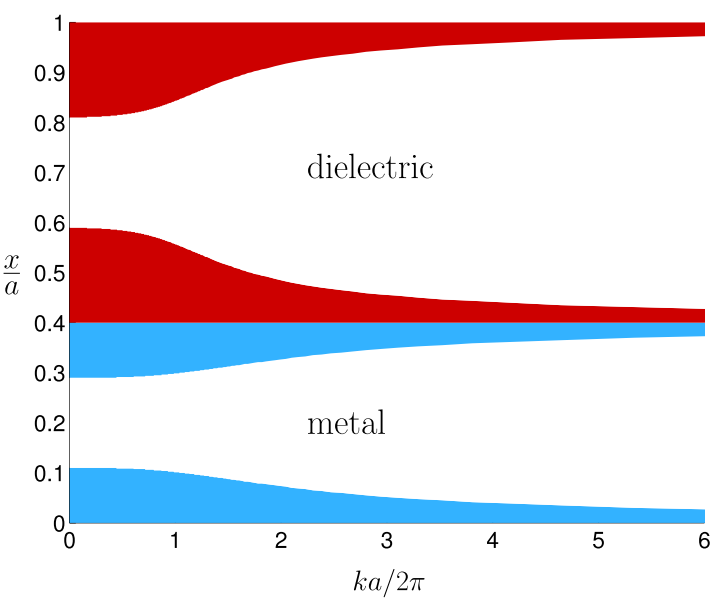

FIG. 5. (Color online) The skin depths versus the axial wave number $k$ for the layer structure. The Bloch wave number is $k_{x}=0$. The skin depth is defined to be the distance from the peak of $E$ to where $E$ decays to $E_{\text {peak }} / e$. The red (dark gray) part is the skin depth in the dielectric part whereas the cyan (light gray) part is the metal part.

$$
\begin{aligned}
& \left.\frac{\partial^{2} E}{\partial x^{2}}\right|_{p, q} \approx \mathcal{L}_{E_{x x}}^{\prime}\left(E_{p-1: p+2, q-1, q+2}, J_{E, r}\right), \\
& \left.\frac{\partial^{2} E}{\partial y^{2}}\right|_{p, q} \approx \mathcal{L}_{E_{y y}}^{\prime}\left(E_{p-1: p+2, q-1, q+2}, J_{E, r}\right),
\end{aligned}
$$

where $\mathcal{L}_{E_{x x}}^{\prime}$ and $\mathcal{L}_{E_{x x}}^{\prime}$ are linear functions. Then the finitedifference scheme for $\nabla^{2} E_{p, q}$ is

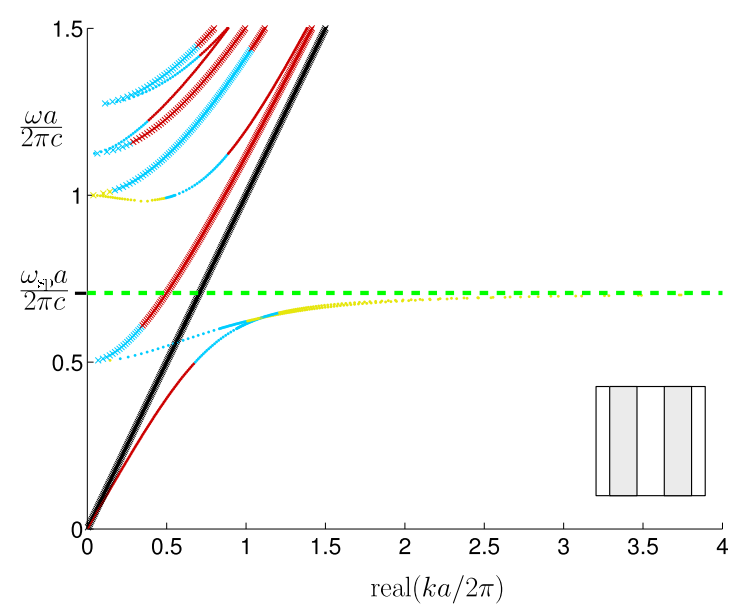

FIG. 6. (Color online) The frequency $\omega$ versus the real part of the axial wave number $k$ for the layer structure with damping. The Bloch wave number is $k_{x}=0$. The corresponding damping frequency is $\frac{\omega_{\tau} a}{2 \pi c}=0.00296$. The solid line is TM mode while the line with cross symbol is TE mode. The imaginary part of $k$ is marked by different colors (grayscale). Red (dark gray dot), cyan (medium dark dot), and yellow (light gray dot) indicate that the imaginary part of $k a / 2 \pi c$ lie in $\left[0,10^{-6}\right),\left[10^{-6}, 10^{-3}\right)$, and $\left[10^{-3}, 10^{-2}\right)$, respectively. 


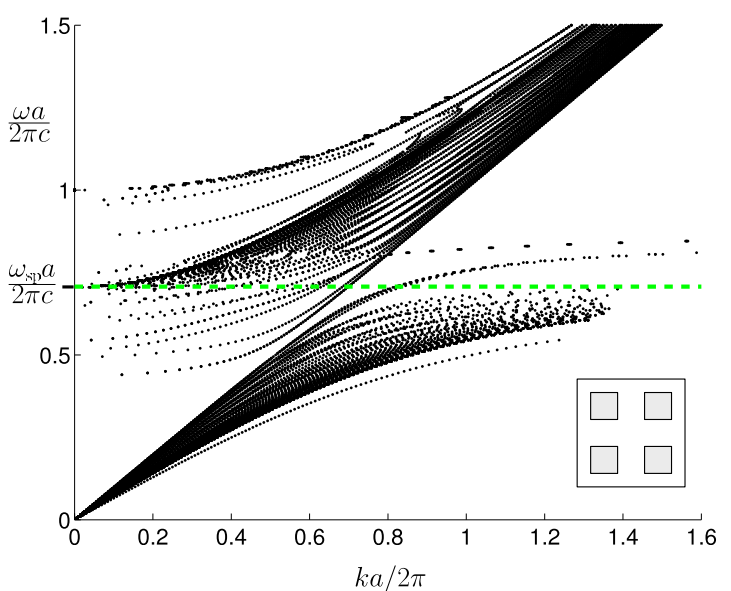

FIG. 7. (Color online) The frequency $\omega$ versus the axial wave number $k$ for the square structure. The Bloch wave vector is $\left(\frac{k_{x} a}{2 \pi}, \frac{k_{y} a}{2 \pi}\right)=(0.5,0.5)$ and the filling ratio of metal is 0.16 .

$$
\nabla^{2} E_{p, q} \approx\left(\mathcal{L}_{E_{x x}}^{\prime}+\mathcal{L}_{E_{y y}}^{\prime}\right)\left(E_{p-1: p+2, q-1, q+2}, J_{E, r}\right) .
$$

Similarly, we can get the finite-difference approximation for $\nabla^{2} H_{p, q}$ :

$$
\nabla^{2} H_{p, q} \approx\left(\mathcal{L}_{H_{x x}}^{\prime}+\mathcal{L}_{H_{y y}}^{\prime}\right)\left(H_{p-1: p+2, q-1, q+2}, J_{H, r}\right) .
$$

\section{Discretize the interface conditions at interface grids}

First, we express the left-hand side of the interface conditions (15) and (16) in terms of the interfacial variables and one-side derivatives:

$$
\left[\frac{1}{\mu} \frac{\partial E}{\partial n}\right]_{R}=\frac{1}{\varepsilon_{+} \mu_{+}} J_{E, r}+\left.\frac{\varepsilon_{-} \mu_{-}-\varepsilon_{+} \mu_{+}}{\varepsilon_{+} \mu_{+} \mu_{-}} \frac{\partial E_{-}}{\partial n}\right|_{R},
$$

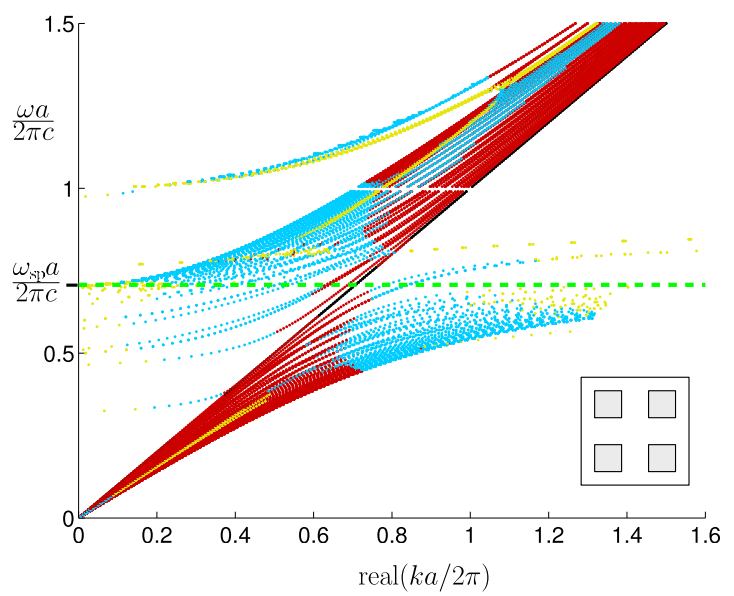

FIG. 8. (Color online) The frequency $\omega$ versus the real part of the axial wave number $k$ for the square structure. The Bloch wave vector is $\left(k_{x}, k_{y}\right)=(0,0)$ and the filling ratio of metal is 0.16 . The corresponding damping frequency is $\frac{\omega_{\tau} a}{2 \pi c}=0.00296$. The imaginary part of $k$ is marked by different colors (grayscale). Red (dark gray dot), cyan (medium dark dot), and yellow (light gray dot) indicate that the imaginary part of $k a / 2 \pi c$ lie in $\left[0,10^{-6}\right),\left[10^{-6}, 10^{-3}\right)$, and $\left[10^{-3}, 10^{-2}\right)$, respectively.

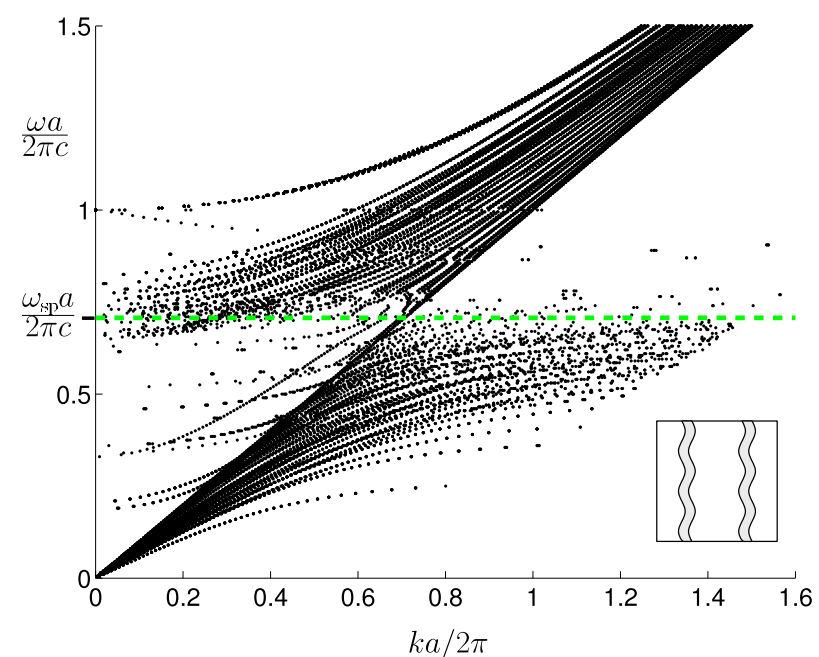

FIG. 9. (Color online) The frequency $\omega$ versus the axial wave number $k$ for the wavy structure. The Bloch wave vector is $\left(k_{x}, k_{y}\right)=(0,0)$ and the filling ratio of metal is 0.16 .

$$
\begin{gathered}
{\left[\frac{1}{\varepsilon \mu} \frac{\partial H}{\partial s}\right]_{R}=\left.\frac{\varepsilon_{-} \mu_{-}-\varepsilon_{+} \mu_{+}}{\varepsilon_{+} \varepsilon_{-} \mu_{+} \mu_{-}} \frac{\partial H_{-}}{\partial s}\right|_{R},} \\
{\left[\frac{1}{\varepsilon} \frac{\partial H}{\partial n}\right]_{R}=\frac{1}{\varepsilon_{+} \mu_{+}} J_{H, r}+\left.\frac{\varepsilon_{-} \mu_{-}-\varepsilon_{+} \mu_{+}}{\mu_{+} \varepsilon_{+} \varepsilon_{-}} \frac{\partial H_{-}}{\partial n}\right|_{R},} \\
{\left[\frac{1}{\varepsilon \mu} \frac{\partial E}{\partial s}\right]_{R}=\left.\frac{\varepsilon_{-} \mu_{-}-\varepsilon_{+} \mu_{+}}{\varepsilon_{+} \varepsilon_{-} \mu_{+} \mu_{-}} \frac{\partial E_{-}}{\partial s}\right|_{R} .}
\end{gathered}
$$

Using Eqs. (43) and (44), we can further express the normal and tangential derivatives $\left.\frac{\partial E_{-}}{\partial n}\right|_{R}$ and $\left.\frac{\partial E_{-}}{\partial s}\right|_{R}$ in terms of grid values of $E$ at nearby Cartesian grids. Then the interfacial

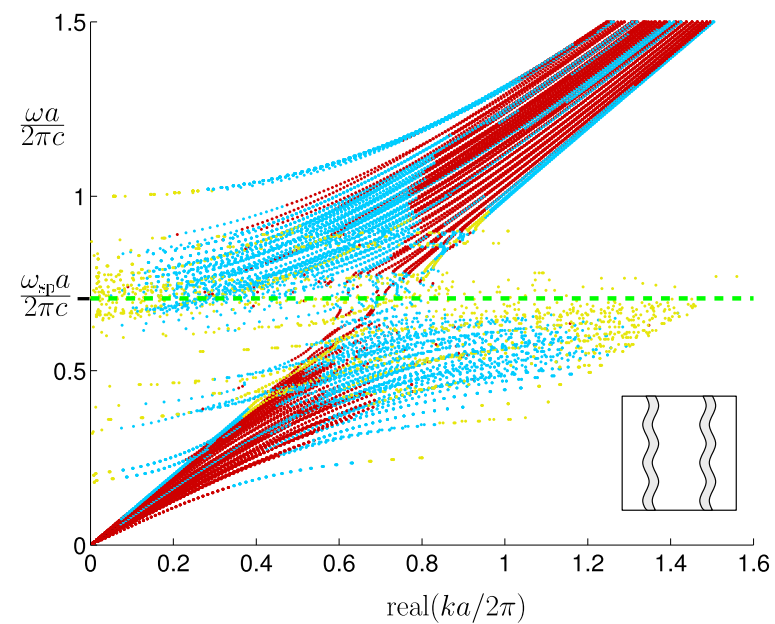

FIG. 10. (Color online) The frequency $\omega$ versus the real part of the axial wave number $k$ for the wavy structure. The Bloch wave vector is $\left(k_{x}, k_{y}\right)=(0,0)$ and the filling ratio of metal is 0.16 . The corresponding damping frequency is $\left(\omega_{\tau} a\right) /(2 \pi c)=0.00296$. The imaginary part of $k$ is marked by different colors (grayscale). Red (dark gray dot), cyan (medium dark dot), and yellow (light gray dot) indicate that the imaginary part of $k a / 2 \pi c$ lie in $\left[0,10^{-6}\right)$, $\left[10^{-6}, 10^{-3}\right)$, and $\left[10^{-3}, 10^{-2}\right)$, respectively. 
operators in Eqs. (15) and (16) are approximated by linear combinations of grid data and interfacial variables:

$$
\begin{gathered}
\Lambda\left[\frac{1}{\mu} \frac{\partial E}{\partial n}\right]_{R} \approx \mathcal{J}_{E_{n}}\left(E_{p-1: p+2, q-1, q+2}, J_{E, r}\right), \\
\frac{\Lambda}{\omega \varepsilon_{0}}\left[\frac{1}{\varepsilon \mu} \frac{\partial H}{\partial s}\right]_{R} \approx \mathcal{J}_{H_{s}}\left(H_{p-1: p+2, q-1, q+2}, J_{H, r}\right), \\
\Lambda\left[\frac{1}{\varepsilon} \frac{\partial H}{\partial n}\right]_{R} \approx \mathcal{J}_{H_{n}}\left(H_{p-1: p+2, q-1, q+2}, J_{H, r}\right), \\
\frac{\Lambda}{\omega \mu_{0}}\left[\frac{1}{\varepsilon \mu} \frac{\partial E}{\partial s}\right]_{R} \approx \mathcal{J}_{E_{s}}\left(E_{p-1: p+2, q-1, q+2}, J_{E, r}\right) .
\end{gathered}
$$

Combining the discretizations for the equations at the Cartesian grids and for the interface condition at the interface grids, we arrive at a quadratic eigenvalue problem:

$$
\mathbf{A}_{\text {mix }} \mathbf{U}_{\text {mix }}+k \mathbf{B}_{\text {mix }} \mathbf{U}_{\text {mix }}=k^{2} \mathbf{U}_{\text {mix }},
$$

where $\mathbf{A}_{\text {mix }}$ and $\mathbf{B}_{\text {mix }}$ are $2\left(N^{2}+N_{\mathrm{J}}\right) \times 2\left(N^{2}+N_{\mathrm{J}}\right)$ matrices and
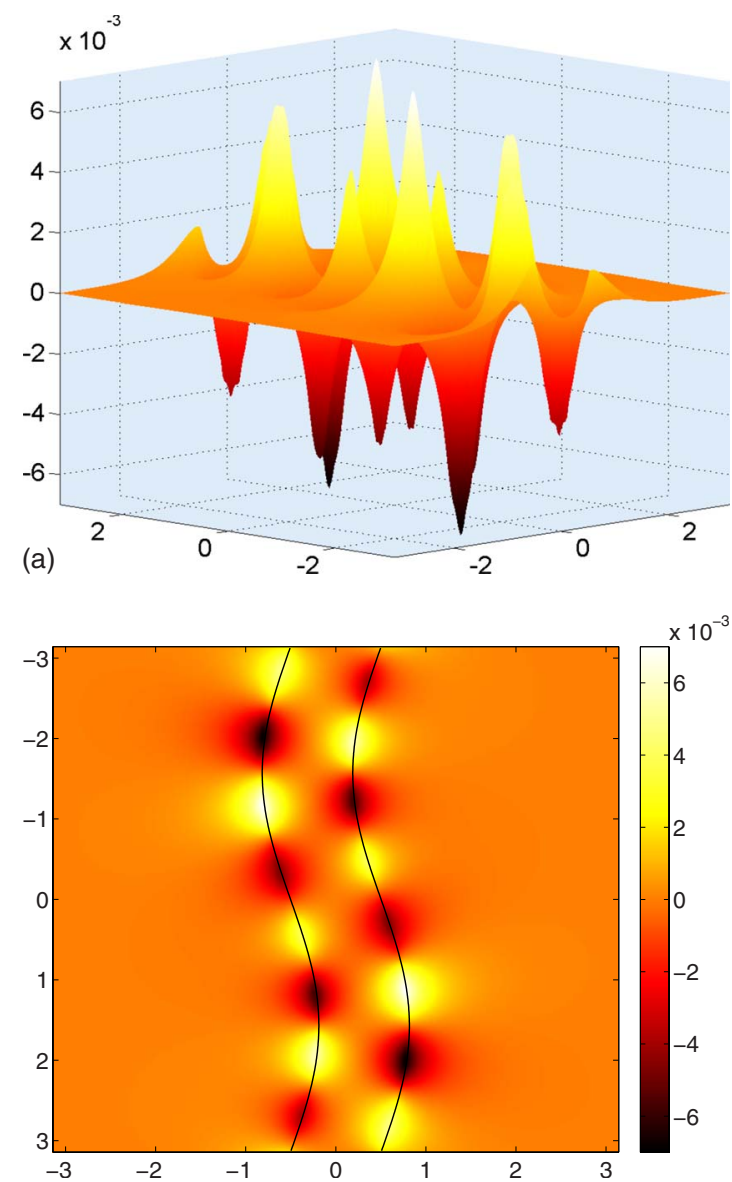

(b)

$$
\mathbf{U}_{\text {mix }}=\left[\bar{E}_{1: N, 1: N}, \bar{H}_{1: N, 1: N}, \bar{J}_{E, 1: N_{\mathrm{J}}}, \bar{J}_{H, 1: N_{\mathrm{J}}}\right]^{T} .
$$

We solve this quadratic eigenvalue problem by doubling the matrix to reduce the problem to an ordinary eigenvalue problem:

$$
\begin{gathered}
{\left[\begin{array}{cc}
\mathbf{0} & \mathbf{I} \\
\mathbf{A} & \mathbf{B}
\end{array}\right]\left[\begin{array}{c}
\mathbf{U}_{\text {mix }} \\
k \mathbf{U}_{\text {mix }}
\end{array}\right]=k\left[\begin{array}{c}
\mathbf{U}_{\text {mix }} \\
k \mathbf{U}_{\text {mix }}
\end{array}\right],} \\
{\left[\begin{array}{cc}
\mathbf{0} & \mathbf{I} \\
\mathbf{A}_{\text {mix }} & \mathbf{B}_{\text {mix }}
\end{array}\right]\left[\begin{array}{c}
\mathbf{U}_{\text {mix }} \\
k \mathbf{U}_{\text {mix }}
\end{array}\right]=k\left[\begin{array}{c}
\mathbf{U}_{\text {mix }} \\
k \mathbf{U}_{\text {mix }}
\end{array}\right],}
\end{gathered}
$$

where $\mathbf{0}$ and $\mathbf{I}$ are zero and identity matrix.

In this formulation, both $\varepsilon$ and $\mu$ are allowed to be arbitrary functions of frequency $\omega$, which can be obtained from either experimental measurement or explicit models. In the Sec. V, we shall adopt the Drude model and solve the above eigenvalue problem for the wave number $k$ in terms of $\omega, k_{x}$, and $k_{y}$.

\section{RESULTS AND DISCUSSION}

The problem to solve plasmonic waveguides can be recast as a dispersion relation in the abstract form $\mathcal{F}\left(k ; \omega, k_{x}, k_{y}\right)$
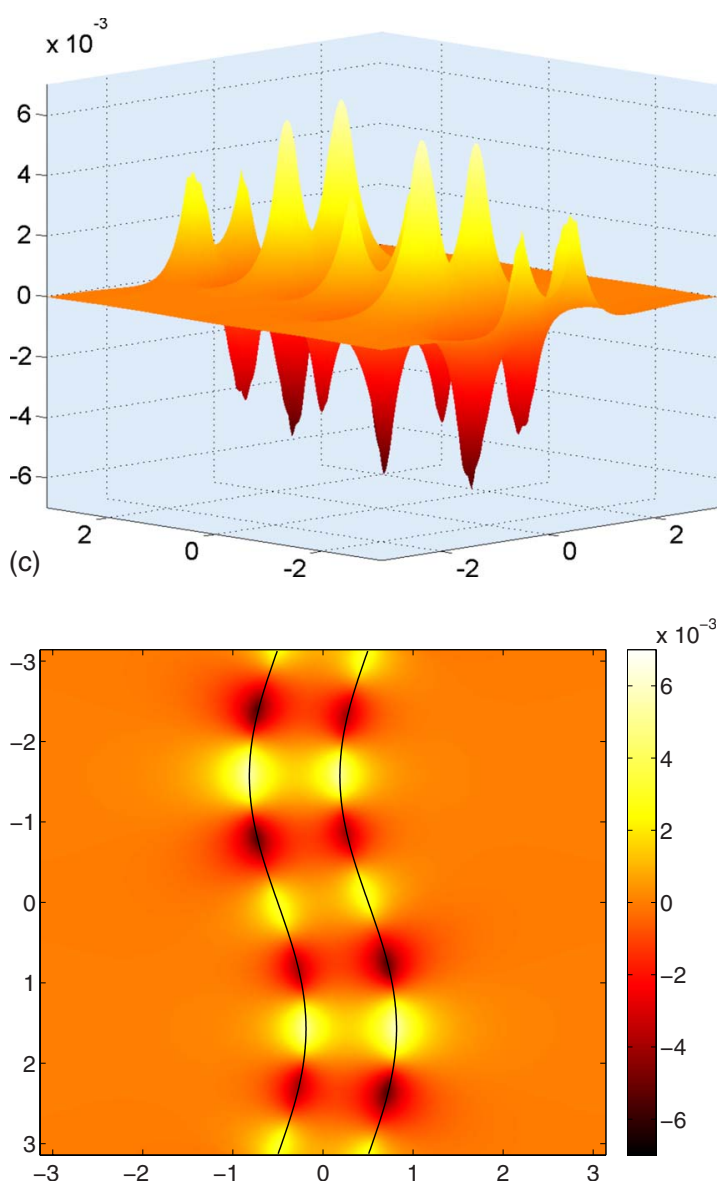

(d)

FIG. 11. (Color online) An eigenmode of the wavy structure. The Bloch wave vector is $\left(k_{x}, k_{y}\right)=(0,0)$ and the filling ratio of metal is 0.16. $(\omega a) /(2 \pi c)=0.7,(k a) /(2 \pi)=0.82815$. (a) and (b): $E / E_{0}$ field; (c) and (d): $H / H_{0}$ field. $E_{0}$ and $H_{0}$ are reference electric and magnetic fields. 


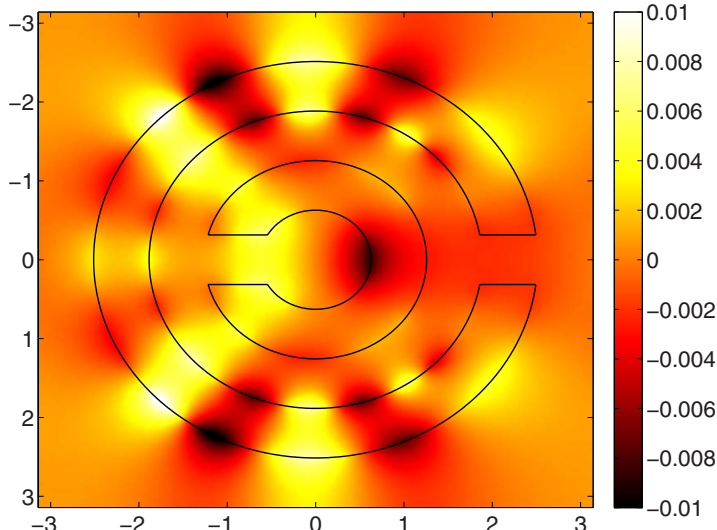

(a)

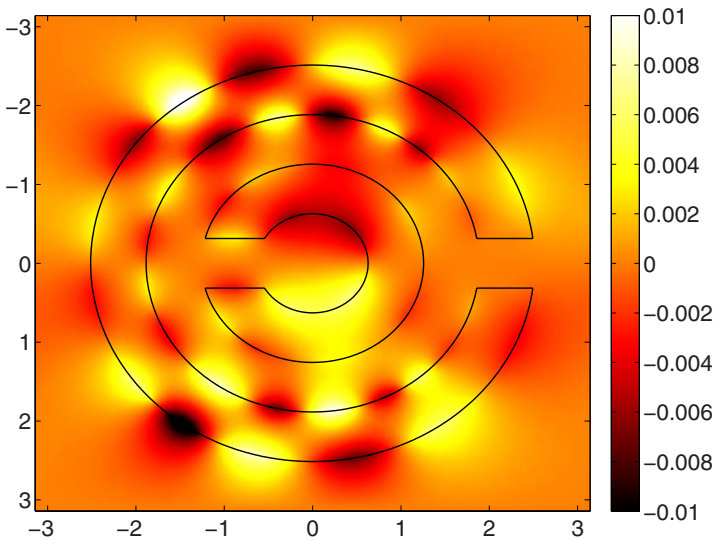

(b)

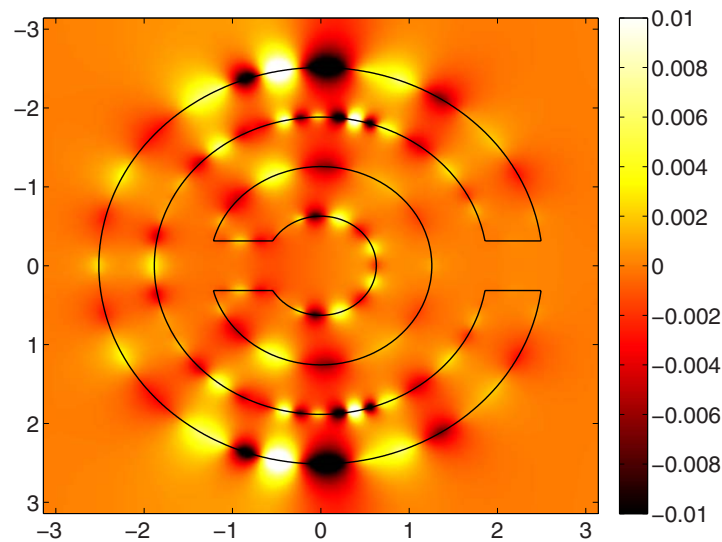

(c)

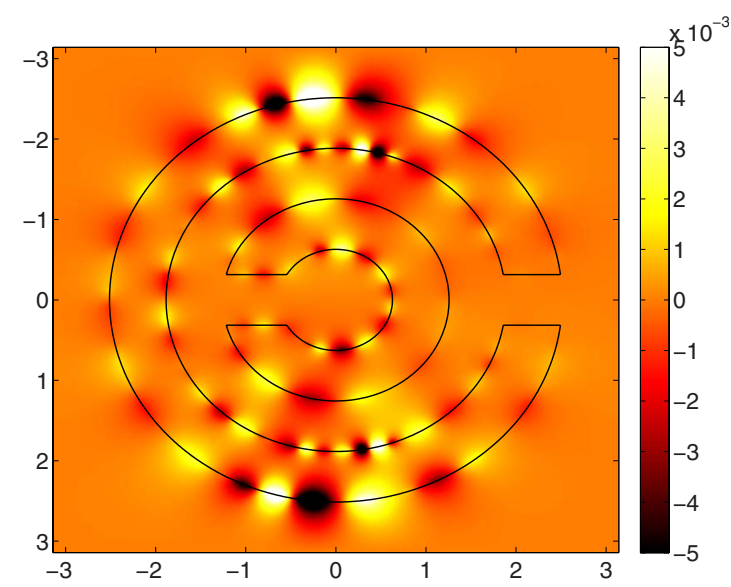

(d)

FIG. 12. (Color online) An eigenmode of the split-ring structure. The interface is plotted as black line. The Bloch wave vector is $\left(k_{x}, k_{y}\right)=(0,0) ;(\omega a) /(2 \pi c)=0.7 ;\left(\omega_{\tau} a\right) /(2 \pi c)=0 .(k a) /(2 \pi)$ is 0.6818 in (a) and (b) and 1.5106 in (c) and (d). $E_{0}$ and $H_{0}$ are reference electric and magnetic fields.

$=0$. In other words, we will solve for the axial wave number $k$ and the associated wave modes with given frequency $\omega$, and planar wave numbers $k_{x}$ and $k_{y}$.

In the present study we consider the Drude model

$$
\varepsilon_{-}=1-\frac{\omega_{p}^{2}}{\omega\left(\omega+i \omega_{\tau}\right)}
$$

for metal, and the host medium is air with $\varepsilon_{+}=1$, and we have chosen $\frac{\omega_{p} a}{2 \pi c}=1$.

We are concerned with signal propagation and energy absorbing problems in plasmonic crystals. Plasmonic waveguides are used to guide surface plasmons (plasmonic signals) along a very thin interface between the metal and host dielectric to realize light propagation in subwavelength metallic structures. ${ }^{2}$ To qualify for this property of light on a wire, the guided wave mode must have very small decay lengths (skin depths) on both sides of the metal-dielectric interfaces, as well as a significantly large group velocity along the axis of propagation.

Figure 4 shows the frequency bands versus the axial wave number for the periodic array of planar metals (without damping). There are two branches below the light line which approach the surface-plasmon frequency $\omega_{\mathrm{sp}}=\frac{\omega_{p}}{\sqrt{1+\varepsilon_{+}}}$for pla- nar interface. These are the propagation modes. The lower branch is more interesting as it has a larger group velocity for $\frac{k a}{2 \pi}<1$. Figure 5 shows the skin depths of this lower branch on the two sides of the metal-dielectric interfaces. These skin depths decrease with increasing the axial wave number, while the corresponding group velocity decreases to zero. For real metal, however, there is one more restriction. Namely, the axial wave number $k$ is no longer real, and its imaginary part measures the length of propagation. Figure 6 shows the frequency bands for the periodic array of planar metals (gold). The corresponding damping frequency is $\frac{\omega_{\tau} a}{2 \pi c}$ $=0.00296$. It is seen that, on the branches below the light line, only the portions close to the light line have longer lengths of propagation. Further, this length of propagation decreases with increasing axial wave number. It is therefore a trade-off problem to choose propagating plasmonic modes in an appropriate range of frequencies.

In contrast to the array of planar metals, the array of square metal cylinders allows more branches of plasmonic modes. Figures 7 and 8 show the frequency bands versus the axial wave number for such a structure without and with damping, respectively. There are many branches of plasmon modes which originate from the origin and form an expansion fan. These various branches allow more freedom for choosing an optimal range of frequencies for propagating 
plasmonic modes. For real metal (8), as before, the length of propagation decreases with increasing axial wave number. In Figs. 7 and 8, above the light line, the dispersion curves cluster around $\omega_{\mathrm{sp}}$ in the limit of $k=0$. These standing plasmonic modes provide a good chance that the periodic structure is an outstanding light-absorbing material near the surface-plasmon frequency at normal incidence to light sources. In real metal, the standing modes suffer damping as seen in Fig. 8. It is noted that the modes closer to the light line either from above or below experience less damping while propagating along the axial direction.

Figures 9 and 10, respectively, show the dispersion relation of a periodic array of wavy metal without/with damping. This example is spectacular as we observe that a wider expanded fan region of plasmon modes below the light line and a significant band broadening near $\omega_{\text {sp }}$ in the limit of $k=0$. This is due to the curvature effect which enables neighboring surface plasmons to interact with each other, and thus lifting certain degeneracy in frequency. For real metal (Fig. 10), again, we note that the modes closer to the light line either from above or below experience less damping while propagating along the axial direction.

Figure 11 shows the $E$ field and $H$ fields of an eigenmode for the wavy structure. It is seen that both $E$ and $H$ fields are concentrated along the wavy edges, roughly in form of isolated islands. For this particular mode, the $[(a)$ and (b) $] E$ field exhibits the mode shape of peak-to-valley structure in the neighboring islands along the two edges, while the [(c) and (d) $] H$ field exhibits the mode shape of peak-to-peak, valley-to-valley structure in the neighboring islands along the two edges. The rich variety of coupling between neighboring islands along the two edges lifts degeneracy of the guided wave modes.

As a final illustration, the method can also be applied to compute plasmonic guided wave modes for split-ring structure, ${ }^{16,17}$ which are often used in negative-index materials. Figure 12 shows the results for two modes with $\mathrm{ka} / 2 \pi$ $=0.6818$ and 1.5106 at the same frequency $\omega a / 2 \pi c=0.7$. Localized plasmons are observed to exist along the edges of the inner and outer rings, but not on the openings except in Fig. 12(a) where the localization of the $E$ field near the opening is not too strong.

\section{CONCLUDING REMARKS}

In the study, we presented the interfacial operator approach with the coupling interface method for solving guided wave modes of plasmonic crystals. It is considered as an eigenvalue problem for the axial wave number. In this formulation, the metallic components can be very general in geometry, and are allowed to have arbitrary frequencydependent permittivity and permeability.

Four metallic shapes are studied: layered, square, wavy and split-ring structures. The Drude model for metal with and without damping is adopted. Except for the layered structure, the plasmonic guided wave modes are in general hybrid modes. Two major conclusions can be drawn. For signal propagation via plasmonic waves, it has been found that with the increasing axial wave number, (i) the group velocity becomes slower, (ii) the skin depth of the plasmonic wave modes becomes thinner, and (iii) the propagation length becomes shorter (if damping effect is considered). These indicate that transmitting signals by plasmonic guided wave is a trade-off problem in choosing an appropriate range of dispersions with less damped effect, thinner thickness, and significant group velocities. In addition, the wavy curvature also broadens the fan structure of the propagation wave modes. This means that the wavy structure provides more frequency modes for signal propagation. For energy absorbing problems, the standing-wave modes (real part of $k \sim 0$ ) are concerned. The curvature in the wavy structure introduces more branches of frequency bands there. This suggests that wavy structure can absorb energy from a wider range of frequency bands. Finally, it is demonstrated that the present method can resolve very fine eigenmodes of the split-ring structure.

\section{ACKNOWLEDGMENTS}

The work was supported in part by the National Science Council of the Republic of China under Contract No. NSC96-2221-E-002-201 and No. NSC96-2628-M-002-017. The authors would also like to thank National Center for Theoretical Science, Taipei Office for partial financial support.

\footnotetext{
*mechang@gate.sinica.edu.tw

${ }^{1}$ R. H. Ritchie, Phys. Rev. 106, 874 (1957).

${ }^{2}$ W. L. Barnes, A. Dereux, and T. W. Ebbesen, Nature (London) 424, 824 (2003).

${ }^{3}$ C. C. Chang, R. L. Chern, C. C. Chang, and R. R. Hwang, Phys. Rev. B 72, 205112 (2005).

${ }^{4}$ R. L. Chern, C. C. Chang, and C. C. Chang, Phys. Rev. B 73, 235123 (2006).

${ }^{5}$ R. L. Chern, C. C. Chang, and C. C. Chang, Phys. Rev. B 74, 155101 (2006).

${ }^{6}$ I. L. Chern and Y. C. Shu, J. Comput. Phys. 225, 2138 (2007).

${ }^{7}$ V. Kuzmiak, A. A. Maradudin, and A. R. McGurn, Phys. Rev. B 55, 4298 (1997).

${ }^{8}$ M. M. Sigalas, C. M. Soukoulis, C. T. Chan, and K. M. Ho, Phys. Rev. B 49, 11080 (1994).
}

\footnotetext{
${ }^{9}$ A. Moroz, J. Phys.: Condens. Matter 6, 171 (1994).

${ }^{10}$ A. Moroz, Phys. Rev. B 51, 2068 (1995).

${ }^{11}$ A. Moroz and A. Tip, Phys. Lett. A 235, 195 (1997).

${ }^{12}$ A. Modinos, N. Stefanou, and V. Yannopapas, Opt. Express 8, 197 (2001).

${ }^{13}$ E. Moreno, D. Erni, and C. Hafner, Phys. Rev. B 65, 155120 (2002).

${ }^{14}$ T. Ito and K. Sakoda, Phys. Rev. B 64, 045117 (2001).

${ }^{15}$ K. C. Huang, P. Bienstman, J. D. Joannopoulos, K. A. Nelson, and S. Fan, Phys. Rev. Lett. 90, 196402 (2003).

${ }^{16}$ S. O'Brien, D. McPeake, S. A. Ramakrishna, and J. B. Pendry, Phys. Rev. B 69, 241101(R) (2004).

${ }^{17}$ J. Zhou, T. Koschny, M. Kafesaki, E. N. Economou, J. B. Pendry, and C. M. Soukoulis, Phys. Rev. Lett. 95, 223902 (2005).
} 